August 12-15, 2012, Chicago, IL, USA

DETC2012-70420

\title{
THE MEANING OF “NEAR” AND “FAR”: THE IMPACT OF STRUCTURING DESIGN DATABASES AND THE EFFECT OF DISTANCE OF ANALOGY ON DESIGN OUTPUT
}

\author{
Katherine Fu \\ Dept. of Mechanical Engineering \\ Carnegie Mellon University \\ Pittsburgh, PA, USA \\ Kenneth Kotovsky \\ Dept. of Psychology \\ Carnegie Mellon University \\ Pittsburgh, PA, USA
}

\author{
Joel Chan \\ Dept. of Psychology \\ University of Pittsburgh \\ Pittsburgh, PA, USA
}

\author{
Christian Schunn \\ Dept. of Psychology \\ University of Pittsburgh \\ Pittsburgh, PA, USA
}

\author{
Jonathan Cagan \\ Dept. of Mechanical Engineering \\ Carnegie Mellon University \\ Pittsburgh, PA, USA
}

\author{
Kristin Wood \\ Eng. and Product Devel. Pillar \\ Singapore Univ. of Tech. Design \\ Singapore, Rep. of Singapore
}

\begin{abstract}
This work lends insight into the meaning and impact of "near" and "far" analogies. A cognitive engineering design study is presented that examines the effect of the distance of analogical design stimuli on design solution generation, and places those findings in context of results from the literature. The work ultimately sheds new light on the impact of analogies in the design process and the significance of their distance from a design problem. In this work, the design repository from which analogical stimuli are chosen is the U.S. patent database, a natural choice, as it is one of the largest and easily accessed catalogued databases of inventions. The "near" and "far" analogical stimuli for this study were chosen based on a structure of patents, created using a combination of Latent Semantic Analysis and a Bayesian based algorithm for discovering structural form, resulting in clusters of patents connected by their relative similarity. The findings of this engineering design study are contextualized with the findings of recent work in design by analogy, by mapping the analogical stimuli used in the earlier work into similar structures along with the patents used in the current study. Doing so allows the discovery of a relationship between all of the stimuli and their relative distance from the design problem. The results confirm that "near" and "far" are relative terms, and depend on the characteristics of the potential stimuli. Further, although the literature has shown that "far" analogical stimuli are more likely to lead to the generation innovative solutions with novel characteristics, there is such a thing as too far. That is, if the stimuli are too distant, they then can become harmful to the design process. Importantly, as well, the data mapping approach to identify analogies works, and is able to impact the effectiveness of the design process. This work has implications not only in the area of finding inspirational designs to use for design by analogy processes in practice, but also for synthesis, or perhaps even unification, of future studies in the field of design by analogy.
\end{abstract}

\section{INTRODUCTION}

Design-by-analogy is a practice in which designers use solutions from other domains in order to gain inspiration or insight for the design problem at hand, and has been shown to be an effective method for inspiring innovative design solutions [1-4]. However, more work is needed to build an understanding of which analogies presented to designers achieve the best design outcomes, as well to develop methods for finding these analogies in an efficient way. Here, designby-analogy is studied from a cognitive perspective, and a patent structuring methodology $[5,6]$ is used to both find analogical stimuli for designers, as well as to analyze the results. The results are compared with previous work to gain a more complete understanding of the discovered effects. First, we review the current understanding of the use of analogy in design, and competing theories regarding the benefits of near vs. far-field analogy in design. Then, we discuss the computational design tools that have been created to facilitate designby-analogy, and the technical foundation of the computational method we use for aiding in the location of these analogies.

\subsection{Design-by-Analogy}

The use of analogy in design has been studied to gain an understanding of how it affects the ideation process and outcomes [712], with some studies specifically examining how the introduction of analogies with different levels of applicability to the design problem affects individual designers [3, 7]. Other work has examined the timing of the introduction of external analogical stimuli within the ideation process, for example by studying the effects of "open goals" [7-9]. Additionally, work has been done to examine the effects of biologically inspired design by analogy on design tasks of solution generation, evaluation, and explanation and analogical methods such as direct transfer, relational abstraction, and problem transformation [13]. The work presented here examines design solution generation based on analogical stimuli, and does not explore the particular methods by which designers might generate or use analogies. 
A large area of exploration in the study of design-by-analogy [1416], and the focus of this work, is analogical distance. In the literature, analogical distance is typically operationalized and studied as a dichotomous variable, with analogies being either near-field or farfield. Near generally means that the analogy is found in the same or similar domain, while far generally means that it is found from a different domain. Further, near-field analogies tend to share a significant number of surface features with the design problem, while far-field tend to share little or none. Far-field analogies, however, can have functional similarities to the design problem that make them apt for analogical transfer. Some literature points to far analogies as being the most promising for creative insights, in which the two domains being compared are very different [17]. Work by Dahl and Moreau showed that the number of far-field analogies used by designers during ideation is positively related to the originality of proposed solutions, as rated by a sample of potential customers [18]. Wilson et al. found that idea novelty increased with exposure to surface dissimilar design examples relative to using no examples, and variety of ideas decreased with exposure to surface similar examples relative to surface dissimilar examples [19]. Often, it is even recommended to use random analogical stimuli, which can perhaps be thought of as "extremely farfield", to make free or wild associations when ideating, as it can encourage wider search of the design space and potentially higher quality ideas as a result [20].

Other literature questions the benefit of far-field analogies [21, 22]. Far-field analogies can be hard to conceive as relevant to a design problem [1], and they can be cognitively challenging to retrieve from memory $[23,24]$. It is evident that a cohesive theory on the effect of analogical distance on design outcomes does not currently exist. One possible reason for this is that there is a lack of consistency and standardization of meaning of the terms "near" and "far," making results difficult to compare. Analogical distance is a continuum rather than a simple dichotomy with categories applied in somewhat arbitrary regions of the continuum; thus, existing studies in the literature may be examining and contrasting different points on the continuum from near to far. It is possible that more than two meaningful levels of distance exist along the continuum, with potentially different patterns of effects at these different points on the continuum. For instance, the potential usefulness of analogical stimuli for supporting creative ideation could vary in a curvilinear fashion from near to far, with very near and very far stimuli being less helpful (e.g., due to low novelty at the near end and low relevance at the far end).

Aside from what kinds of analogies are most beneficial and stimulating for designers, it remains unclear how to find these analogies in an efficient or automatic way. There have been methodologies developed to employ design-by-analogy, however, including Synectics [25]- group design through analogy types; French's work on inspiration from nature [26]; a semantic active verb mapping of design problems known as the WordTree method [27-29]; Biomimetic concept generation [30]- a systematic tool to index biological phenomena that links to textbook information; and analogous design using the Function and Flow Basis [31, 32]analogous and nonobvious product exploration using the functional and flow basis. Though these "pen-and-paper" and semi-automated methods can be beneficial, they can also be cumbersome or inefficient, highlighting one of the major benefits of computational design tools, reviewed next.

\subsection{Computational Design Tools}

Computational design tools provide a promising way of efficiently and automatically finding analogical stimuli with which to inspire designers. A natural source for analogical stimuli is the U.S. patent database, which is the source of analogies for the work presented here, as well as a great deal of other research, including TRIZ [33] using heuristic rules to help engineers overcome impasses in functional reasoning by searching through patents; an axiomatic conceptual design tool [34] combining TRIZ and functional basis; patent mining [35-37] characterizing them by citations, claims, average number of words per claim, number of classes that the patent spans, etc.; design repository work incorporating function-based search using Chi Matrix and Morphological Matrix techniques [38]; PatViz [39], allowing for visual exploration of iterative and complex patent searches and queries using all types of patent data, including full text, which relies on structures that are either predefined or userdefined classification schemes; patent database search using a mapped functional basis [40]; a BioMedical Patent Semantic Web [41] finding semantic associations between biological terms within biomedical patent abstracts and returning a ranked list of patent resources and a fully connected Semantic Web that displays the relationships between the important terms and between resources; and a topic model based taxonomy or hierarchical structure only used to categorize the remaining documents into "topics" [42]. Our work is differentiated from these efforts in that it focuses on using the full textual content of the patents, which it is hoped will allow for richer outcomes, on structuring design repositories and more open ended analogical transfer, and on multiple structure types generated using a hierarchical Bayesian algorithm.

The size and complexity of the U.S. patent database presents considerable challenges to making it useful to designers. The methodology for structuring patents, first presented by $\mathrm{Fu}$ et al. in $2011[5,6]$, and briefly reviewed here, enables the extraction of their interrelatedness and interconnectedness. Ultimately, designers might be able to use these structures to strategically choose analogical stimuli to expose themselves to, or even traverse and explore the space in a more intentional and meaningful way. Designers have the potential to create more innovative designs with more efficient and insightful access to analogical stimuli. We delve into the first steps of using these structures in this manner by testing their output with a cognitive engineering design study, to be described in Section 2.3. The method and algorithm for creating these structures is presented next.

\subsection{Discovering Structural Form}

There is a history of describing human cognition using Bayesian models [43], and this link between the Bayesian algorithm and human cognition is a main motivation for choosing this algorithm. The closer the output is to human conceptualization of the data or information, the more easily understood and useful it will be to humans in design practice.

Kemp and Tenenbaum [44] use Bayes Rule to calculate the probability that the data has structure $\mathrm{S}$ and form $\mathrm{F}$ given data $\mathrm{D}$. A form is defined by the graph grammar that is used to create it. These forms, including a partition, chain, order, ring, tree, hierarchy, grid, and cylinder, and their associated graph grammars, are described in more detail in the source literature [44]. These structures originate from psychology literature [45] and appear in formal models in many different research efforts [46-57]. Kemp and Tenenbaum argue that the structural forms included in the algorithm are often and commonly found, are "useful for describing the world, and that they spring to mind naturally when scientists seek formal descriptions of a domain" [44]. Structures have been used successfully to uncover previously unconsidered or unknown relationships in biology and chemistry - i.e., Linneaus' discovery of the tree structure that best describes the relationships between living organisms, or Mendeleev's periodic structure of elements [44]. The Bayesian algorithm for discovery of 
structure in the patent database can facilitate a meaningful exploration of potentially analogous solutions, and potentially stimulate the discovery of useful, perhaps previously unconsidered, relationships and functional solutions by designers.

A structure $S$ is a particular instantiation of a form $F$. To be clear, a graph of data $D$ with a certain form can be represented by a number of different configurations, or structures. The three terms that go into calculating this posterior probability, which serves as the score of a particular structural form within the algorithm, were chosen and calculated as follows [43]: $P(S, F \mid D) \propto P(D \mid S) P(S \mid F) P(F)$, where:

1. $P(F)$, the prior on the space of forms, is a uniform distribution over the forms under consideration.

2. $P(S \mid F)$, the prior on the structures, favors graphs where $k$, the number of clusters, is small: $P(S \mid F) \alpha \theta^{k}$ if $S$ is compatible with $F$, and $P(S \mid F)=0$ otherwise; here, $\theta=\mathrm{e}^{-3}$.

3. $P(D \mid S)$, the likelihood, measures how well the structure $S$ accounts for the data $D . \quad P(D \mid S)$ will be high if the features in $D$ vary smoothly over the graph $S$, that is, if entities nearby in $S$ tend to have similar feature values.

4. The normalizing constant, the marginal probability, is calculated using set theory, as a sum of the products of the number of $\mathrm{F}$ structures with $\mathrm{k}$ occupied cluster nodes and the number of ways to partition $\mathrm{n}$ elements into $\mathrm{k}$ nonempty sets.

\subsection{Latent Semantic Analysis}

Latent Semantic Analysis (LSA) is used to pre-process design document texts (here, patent texts) in order to extract the contextual similarity of documents [58]. LSA has been successfully used in studies of communication among designers [59, 60] and functional content in engineers' descriptions of devices [61]. The output is a similarity matrix [62-64], which serves as the input to Kemp and Tenenbaum's algorithm. LSA has four main steps:

1. Creating a word-by-document matrix, in which the columns represent the documents, the row represent the words within the documents, and the cells are populated by the number of times each word appears in each document.

2. Performing an "entropy weighting", which is a two part transformation on the word-by-document matrix that gives a more accurate weighting of the word-type occurrences based on their inferred importance in the passages.

3. Performing Singular value decomposition (SVD) on the transformed word-by-document matrix, with an output of the $\mathrm{U}, \mathrm{S}$, and $\mathrm{V}$ matrices.

4. Calculating the cosine similarity between documents by multiplying $\mathrm{S}$ and the transpose of $\mathrm{V}$ and calculating the dot product between all pairs of resulting vectors, yielding a similarity matrix for the documents [62-64].

\section{METHODOLOGY}

This study serves (1) as a validation of the structuring of patents generated using LSA and the Kemp and Tenenbaum algorithm for discovering structural form and (2) as an examination of the effect of "near" and "far" external analogical stimuli on design output quality. The first part of the methodology section describes the steps taken to generate the structure of patents, while the second part details the set up and process by which the cognitive engineering design study was performed.

\subsection{Generating Structures}

\subsubsection{Choosing Initial Patent Set}

A random number generator was used to create a list of random patent numbers, from which a subset of 45 patents was chosen that were classified within the U.S. Patent classification system as "Body Treatment And Care, Heating And Cooling, Material Handling And Treatment, Mechanical Manufacturing, Mechanical Power, Static, and Related Arts". The full text of these 45 random "mechanical" patents were used in the next steps in Section 2.1 to generate the structure used to choose the analogical stimuli for the cognitive experiment.

\subsubsection{LSA Preprocessing}

In order to extract the relationships between the patents in the set, the contextual similarity of the patents was evaluated through the use of LSA. This contextual similarity took the form of a symmetric similarity matrix populated by pairwise cosine similarity values describing the level of semantic similarity between any two patent documents. The full text, including the abstract and description of each of the 45 patents was run through a part-of-speech tagger, in which the verbs, adverbs, adjectives, and nouns are tagged separately, including repeated words. In this application, the part-of-speech tagger served to remove any non-words from the documents. LSA as described in Section 1.4 was then run on the set of 45 full text part-ofspeech tagged patents. The resulting cosine similarity matrix served as the input data to the algorithm for discovering structural form. It is important to recognize that this data is not unlike the similarity data used in Kemp and Tenenbaum's work [44]. This LSA pre-processing step does not change the functionality of the algorithm devised by Kemp and Tenenbaum, though it does serve as a novel way of generating similarity for input to the algorithm.

\subsubsection{Structural Form Discovery}

Kemp and Tenenbaum's algorithm for discovering structural form as it was applied to the output data described in Section 2.1.2 involves the following steps $[44,65]$ :

1. The similarity data $D$ was preprocessed by shifting the mean of the matrix to zero. The normalized covariance matrix for $D$ was calculated, defined as $(1 / \mathrm{m}) D D^{\mathrm{T}}$, where $\mathrm{m}$ is the number of features, or non-redundant non-trivial words included in the entire set of patents. Shifting the mean of $D$ to zero normalizes the feature matrix to allow the calculated covariance to be comparable to the "empirical covariance."

2. The form $F$ and the structure $S$ of that form that best capture the relationships between the set of 45 patents was found by maximizing the posterior probability - the probability that the data has structure $\mathrm{S}$ and form $\mathrm{F}$ given data $\mathrm{D}$; i.e., search for the structure $S$ and form $F$ that jointly maximize the scoring function $P(S, F \mid D)$. For example, the particular set of 45 patents in this study might be best described by the structural form of a grid, or perhaps as a hierarchy or other structure.

3. A separate greedy search was run for each of the eight candidate forms, in order to identify the structure and form that maximize the posterior probability:

- All 45 patents were assigned to a single cluster.

- The algorithm split a cluster at each iteration, using a graph grammar that builds the structure (i.e., grid) after each split.

- The algorithm attempted to improve the score (the posterior probability) using several proposals, including proposals that move an entity from one cluster to another and proposals that swap two clusters.

- The search concluded when the score could no longer be improved. 
The eight forms explored with the algorithm were the partition, chain, order, ring, tree, hierarchy, grid, and cylinder. More detail on these forms and their generative grammars can be found in the source literature of Kemp and Tenenbaum [44, 65]. These forms were used as candidate forms. The output of this step of the methodology is the best structure (instantiation) of each candidate form, and the associated posterior probability. The best structure of the set of eight can be judged by which has the highest posterior probability value.

\subsection{Choosing Varying Stimulus Set}

The main thrusts of the experiment were to (1) validate the use of structuring patents as an automatic analogical inspiration generator, and (2) explore, using the structure of patents, how analogical stimuli of different levels of distance from the design problem would affect the design output quality. In order to achieve these two goals, the winning structure generated in the previous step was a hierarchy, and was used to choose 5 patents that were "near" and 5 patents that were "far" from the design problem description in the structure. The location of the design problem description, or perhaps "starting point", was determined by calculating its semantic similarity to each cluster in the structure and choosing the node with the highest similarity. Near patents were chosen from nodes 0 to 1 node away from the design problem description location. Far patents were chosen from nodes 3 away from the design problem description. The five near and five far patents served as the varying stimulus set for the cognitive engineering design study described in Section 2.3. The structure is presented as Figure 4 in the Results Section.

\subsection{Experimental Procedure}

\subsubsection{Participants}

This study was performed at the University of Texas at Austin, USA with students enrolled at the university. There were 72 participants total, two of which were not included in the analysis and results due to lack of complete participation. There were 10 graduate level participants, and 62 undergraduate level participants. Seventeen participants were female, 52 participants were male, and 3 participants did not indicate their gender. All participants had adequate domain knowledge of engineering and all but 2 had at least some design experience, consisting of some combination of course-related design projects, industry experience, and structured design courses and training in design tools. Detailed in the next step, there were 24 participants in the "Near" condition, 24 participants in the "Far" condition, and 24 participants in the Control condition.

\subsubsection{Conditions}

The independent variable was the patent distance in structure measured from design problem description position. There were three conditions:

1. "Near" Patents - These patents were zero or one node away from where the design problem laid in structure. The varying stimulus set included 5 near patents, with each participant exposed to some combination of 3 of the 5 .

2. "Far" Patents - These patents were three nodes away from where the design problem laid in structure. The varying stimulus set included 5 near patents, with each participant exposed to some combination of 3 of the 5 .

3. Control - This condition of participants received no external stimulus during the experiment.

\subsubsection{Design Problem}

The design problem given to the participants to test the effect of the external analogical stimuli on the design quality output was to design a low cost, easy to manufacture, and portable device to collect energy from human motion for use in developing and impoverished rural communities, e.g., India, many African countries. The design problem description as supplied to the participants is as follows:

Design a device to collect energy from human motion for use in developing and impoverished rural communities in places like India and many African countries. Our goal is to build a low-cost, easy to manufacture device targeted at individuals and small households to provide energy to be stored in a rechargeable battery with approximately $80 \%$ efficiency. The energy is intended to be used by small, low power draw electrical devices, such as a radio or lighting device, hopefully leading to an increase in the quality of life of the communities by increasing productivity, connection to the outside world, etc. The target energy production is 1 $k W$-hour per day, roughly enough to power eight 25 Watt compact florescent light bulbs for 5 hours each per day, or enough to power a CB radio for the entire day.

For reference, an average adult human can output about 200 watts with full body physical activity for short periods of time, with a significant reduction for sustained power output.

This design problem has been used in previous work to examine design by analogy [66]. As in the previous work, the problem was chosen to be challenging and meaningful to the participants. The lack of known or successfully implemented solutions make this design problem appropriately challenging, yet it is sufficiently simple such that meaningful design ideation could begin immediately. The problem is judged as meaningful due to its social impact and relevancy to current efforts in the engineering and product design fields, encouraging an appropriate level of engagement from the students during ideation [67-69].

\subsubsection{Materials}

Each participant received an envelope at the outset of the study, which included:

- Consent form

- Design problem description

- Three patents, one to a page, including the abstract and one key diagram from each (these were not included in Control condition envelopes)

- One stapled packet including:

- Instructions for Phases A, B, and C

- Ideation box sheets for Phases A and C

o Post-experiment survey

$\circ$ Demographics survey

\subsubsection{Cognitive Study Procedure}

The study was run in three separate sections, one 10 -student graduate class, and two undergraduate classes with 39 and 23 students. There were three phases in the study. Phase A was a pre-stimuli ideation phase, in which participants in all conditions worked for 10 minutes on the design problem described in Section 2.3.3. In Phase B, participants in the "Near" and "Far" conditions had 5 minutes to read and understand three patents from their respective varying stimulus sets. The Control condition participants continued to ideate during Phase B. In Phase C, all participants returned to ideating on the given design problem.

Envelopes containing the materials listed in Section 2.3.4 were distributed in a random manner by condition and geographic location in the room for all three sections of the study. Participants signed consent forms, and were told the overall timeline of the study. They then completed the three phases described above, and were given one minute warnings before each phase was over. The experimenter announced that Phase $\mathrm{C}$ had concluded after 15 minutes had elapsed. 
The participants were then asked to complete the debriefing survey and the demographics survey. After they completed the two surveys, they were asked to place all materials back into the envelope in which they came and return the envelope to the experimenter.

\section{DESIGN METRICS}

The design output from the participant was expressed on paper provided to the participants. The paper had two boxes per page, in which participants were instructed to include one idea per box. Each idea included either textual description of the concept, a sketch of the concept, or the majority of the time, both a textual description and sketch. To fully understand the effects of the inspirational stimuli on the participants' ideation, participant-generated ideas were coded for a range of design metrics. The following section details the metrics used to understand the design output and performance of the participants.

\subsection{Design Evaluation Metrics}

To explore the effect of the external inspirational stimuli of different analogical distances on design quality output of individuals, several design evaluation metrics were used. For consistency and comparability, these metrics are the same as those used in our previous work with design by analogy with minor modifications noted below [66]. The metrics used were: (1) quantity of ideas, (2) breadth of search through the design solution space, (3) novelty of ideas, and (4) quality of ideas. Quantity and breadth were used to examine how the participants were ideating; quantity of ideas was meant to give a sense of whether participants were generating and/or refining a small number of ideas or exploring larger numbers of concepts and variations on these concepts, the latter of which is correlated with a greater likelihood of higher quality ideas [64]; breadth of search was intended to gauge participants' ability to come up with a wide variety of ideas, a skill found to correlate with the ability to restructure problems - arguably a vital skill in design problem solving [70-72]. The novelty and quality metrics focused on the design output of the participants. Quality of design output was measured because it is most important that design solutions meet customer requirements. A design might be novel, but if it does not meet customer needs or specifications, it is not an acceptable solution to the problem [70]. Novelty was examined due to the general consensus in the literature that creative products are at least novel $[70,71]$.

\subsection{Quantity}

Quantity of ideation was defined as the number of solution concepts generated after receiving examples, i.e., from the Phase $\mathrm{C}$ of the experiment, that met the minimum constraints of the design problem, namely (1) the device generates electricity, and (2) it uses human motion as the primary input. To account for effects of individual differences in quantity of ideation and focus on the effects of examples, analyses adjusted for the number of solution concepts generated in the first phase, which acted as a covariate to adjust for baseline variation in quantity across participants.

\subsection{Breadth}

Breadth was conceptualized as the proportion of the space of possible solutions searched by a given participant. The space was defined by using functional decomposition to create a set of possible subfunctions of solutions to the design problem, based on the methodology and consistent with those used in our previous design by analogy study [66] informed by the function and flow basis of Hirtz et al. [32]. The original set of subfunctions was the same as that used in the previous study [66]. The subfunctions were divided into two parts - how and what; how signifies the component that implements the subfunction, and what signifies either the input or output flow of the subfunction, whichever is less specified. For example, for the subfunction "import human", the how might be "crank" and the flow might be "hand." The initial set of solutions, or possible instantiations of each subfunction, was re-used from the previous design by analogy work [66], and a few were added if they were not already in the preexisting solution space. A doctoral candidate in mechanical engineering coded the solutions to the subfunctions for each idea, and a second mechanical engineering doctoral candidate independently coded $25 \%$ of the data for inter-rater agreement. Agreement was assessed at two levels: the first level assessed the degree to which the two coders agreed whether or not an idea provided a solution to a given sub-function; the second level assessed the agreement between coders on the type of solution to the sub-function, given that they had agreed a solution was provided. Agreement on the first level was excellent (Cohen's $\kappa=.94)$, and agreement on the second level was acceptable ( $\kappa=.74$ averaged across sub-functions).

As in the previous work, only a small subset of the subfunctions that were coded were common enough for calculated estimates of breadth, novelty, and diversity to be stable (i.e., base rate greater than 0.1 , collapsed across conditions):

1. Import human energy (how)

Import/accept (what) human interaction

2. Transform human energy to mechanical energy (how)

Transform human energy to (what) mechanical energy

3. Import other energy source (how)

Import (what) other energy source

4. Transform other energy source to mechanical energy (how)

Transform other energy source to (what) mech. energy

5. Transform mechanical energy to electrical energy (how)

Transform (what) mechanical energy to electrical energy

Similar to the previous work, there were only two solution types in the subfunction of "store electrical energy," (battery and capacitor), and the frequency of occurrence of these two solutions were nearly equal, and therefore would not have significant impact on differentiating between participants in terms of breadth or novelty calculations.

We defined the space of possible solutions for each of the what and how components of each sub-function by enumerating the number of distinct solution types generated by participants across the final phase of ideation. A breadth score $b_{j}$ for each participant on subfunction $j$ was given by:

$$
b_{j}=\sum_{k=1}^{n} w_{j k} \times \frac{C_{j k}}{\mathrm{~T}_{j k}}
$$

where $C_{j k}$ is the total number of solution types generated by the participant for level $k$ of sub-function $j, T_{j k}$ is the total number of solution types produced by all participants for level $k$ of sub-function $j$, and $w_{k}$ is the weight assigned level $k$. To give priority to breadth of search in the what space (types of energy/material manipulated), we gave a weight of 0.66 to the what level (which was assigned to $k=1$ ), and a weight of 0.33 to the how level (which was assigned to $k=2$ ). An overall breadth score for each participant was given by the average of breadth scores for each of the three sub-functions $j$.

\subsection{Novelty}

Each idea's novelty score was given by: 


$$
\frac{1}{i} \times \sum_{i} \frac{\sum_{j} w_{j} \times R}{1}
$$

where $R$ is the rarity score for the idea's solution for the $j^{\text {th }}$

component of the $i^{\text {th }}$ subfunction. The overall rarity score for the $i^{\text {th }}$ sub-function is given by the weighted average of the rarity scores for the $j$ components of the subfunction, with $w_{j}$ as the weight for each component. All $j$ weights summed to 1 . The unweighted average of rarity scores for all $i$ subfunctions constituted the aggregate novelty score for each idea. [72]:

$R$ for each solution was computed using a formula adapted from

$$
\frac{T-C}{T}
$$

where $T$ is the total number of solution tokens for a given component of a given subfunction in a baseline solution space, and $C$ is the number of solutions tokens of the same type as the current solution. This baseline space included only solution types from a universe of 583 ideas generated by participants who either had or had not received patent examples, including ideas from participants in a previous ideation study using the same design problem [66]. Solution types that did not occur in this space were automatically set to $R=1$. This metric design allowed for example-inspired novel solutions shared by participants who saw the same example to still be counted as novel, assuming that these solution types were unlikely to be generated in the absence of the example, and therefore unlikely to be represented in the baseline solution space.

Recall that each idea was coded for functionality in terms of its how and what for a set of given subfunctions. For purposes of computing $R$, each subfunction had three components: a description of (1) the type of flow, or the what (e.g., energy, human body part) being acted upon, (2) the type of instantiated device function, or the how (e.g., pedals, piezoelectric plate) acting upon the flow, and (3) a description combining the function (how) and flow (what) into a compound function statement. For example, one common idea was transforming human pedaling action into rotational energy, which is then transformed into electrical energy via a dynamo-type generator. For the subfunction "import human energy/interaction", the what for this solution would be "foot", the how would be "pedals", and the compound statement would be "foot with pedals".

The flow (what), function (how), and compound components for each subfunction were given weights of $0.5,0.3$, and 0.2 , respectively. These weights were chosen to reflect the order of "difficulty" in achieving rarity. The variety of flows (whats) tends to be more limited (e.g., most ideas transform human energy into rotational mechanical energy); thus, rarity should count for more in this space. However, in the compound space, the likelihood of finding two ideas with the exact same combination of flow (what) and function (how) is much lower, thereby lowering the threshold for rarity; thus, rarity should count for less in this space.

\subsection{Quality}

The quality of solution concepts was determined by first selecting the best concept from each phase for each participant across all phases. This coding was done by a doctoral candidate in mechanical engineering. Twenty-five percent of the data was independently coded by a second doctoral candidate in mechanical engineering for interrater reliability, which was found to be at an acceptable level of $74 \%$ agreement. These "best" concepts were then evaluated using a detailed quality analysis, similar to that which was used in our previous design by analogy work [66]. This analysis involved measuring quality on a set of subdimensions, in a Pugh chart type of format. The subdimensions, corresponding to a set of projected customer requirements, were as follows:

1. Cost

2. Feasibility of materials/cost/manufacturing

3. Feasibility of energy input/output ratio

4. Number of people required to operate device at a given moment

5. Estimated energy output

6. Portability

7. Time to set up and build, assuming all parts already available at hand

The subdimensions were generated by the first author, a doctoral candidate in mechanical engineering with a research focus in design methods, and were checked for validity by two other authors who are mechanical engineering faculty in the field of engineering design. The solution concepts were scored on each of the subdimensions on a 5point scale ranging from 0 to 4 , where 0 is unacceptable and 4 is excellent. The judgment of the score for a concept in each subdimension was explicitly described prior to rating the designs. Inter-rater reliability for the first and last sub-dimensions (cost and time to set up and build) was unacceptable $(\mathrm{r}<.2)$, so these dimensions were dropped. Reliability averaged across the remaining sub-dimensions was acceptable at $r=.52$.

The overall quality score for each solution for the detailed quality analysis was computed using the following formula:

$$
Q=\frac{\sum_{j=1}^{n} q_{j} \times r_{j}}{Q_{\max }}
$$

where $q_{j}$ is the quality score for quality subdimension $j, r_{j}$ is the reliability of the coding for that subdimension, and $\mathrm{Q}_{\max }$ is the maximum possible overall quality score, which would be given by setting $\mathrm{q}_{\mathrm{j}}$ to 4 for each subdimension. The subdimensions were weighted by reliability as judged by the raters to minimize the influence of measurement error. The overall quality score for the detailed analysis was a proportion of the maximum possible quality score, yielding values between 0 and 1 .

\section{RESULTS}

Separate analyses of variance were performed for each of the metrics described in the previous section. Bonferroni corrections were applied to all post-hoc contrasts to correct for Type I error inflation (false alarms) due to multiple comparisons. No significant effects were seen across conditions in quantity $(F(2,67)=0.05, p=0.94)$ or breadth of ideas $(F(2,61)=0.47, p=0.62$.

In novelty for the current study, we examined both mean novelty and maximum novelty. The independent variable of distance of analogical stimuli had a significant effect on maximum novelty of ideas, $(F(2,59)=4.139, p=0.02)$. Post-hoc pairwise comparisons showed that the most novel concepts generated by participants in the far condition were, on average, significantly less novel compared to concepts generated by participants in either the near (Cohen's $d=-$ $0.82, p=0.02)$ or control conditions $(d=-0.91, p=0.008$; see Figure 1 , right). There was no significant effect on mean novelty $(F(2,61)=$ 1.626, $p=0.20$ ), but the mean trends were similar to those in maximum novelty, where the far condition achieved less novelty than the near and control conditions. These results are contradictory to those found in our previous work, which stated that "far" analogical stimuli are correlated with higher maximum novelty. This discrepancy will be discussed in detail in the Discussion Section. Novelty results from the previous study and the current study are depicted in on the left in Figure 1. 
A significant effect of distance of analogical stimuli on mean quality was found $(F(2,65)=4.07, p=0.02)$. In post-hoc pairwise contrasts, it was found that the near condition had significantly higher quality design output than the far condition $(d=0.76, p=0.02)$, and was trending (though not significantly) toward higher quality design output than the control condition $(d=0.54, p=0.23)$.

In addition, in the current study, a significant effect of distance of analogical stimuli on maximum quality was found $(F(2,65)=4.13, p$ $=0.02$; see Figure 2 right). Post-hoc contrasts showed that the highest quality concepts generated by participants in the far condition were, on average, of significantly lower quality compared to concepts generated in the near condition $(d=-0.76, p=0.02)$, and trending (though not significantly) towards worse quality than the control condition $(d=-0.72, p=0.11)$. As with novelty, these results are contrary to those found in our previous work, which states that far analogical stimuli have a positive, albeit indirect, effect on quality: the

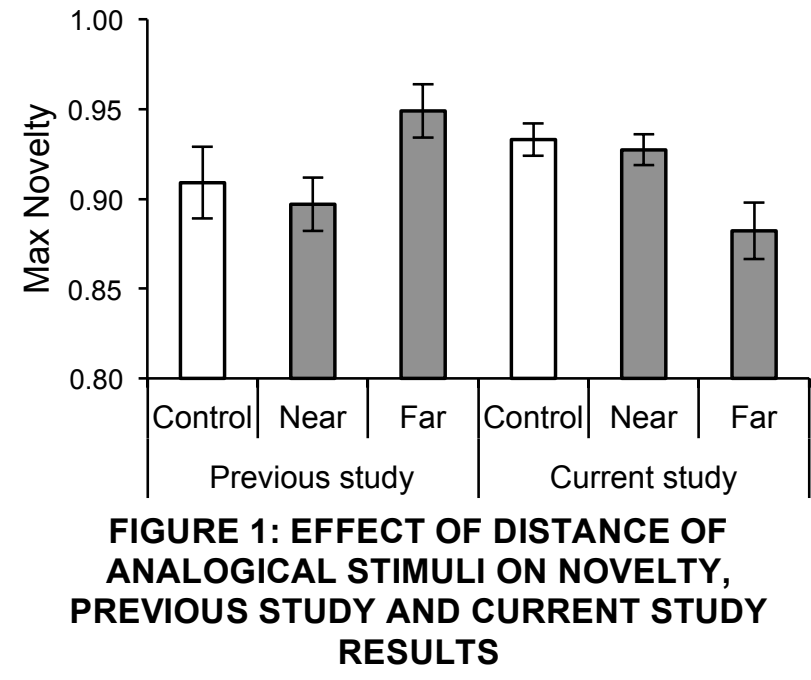

far stimuli resulted in higher variability in quality of ideas [68], which was positively associated with maximum quality (in accord with other findings in the literature $[66,73])$. Variability of quality was not calculated in the current study because we only coded the best concepts from each participant. The discrepancy between the quality results in the current study and the previous study will be discussed in depth in the next section.

One final result concerned the debriefing survey administered to the participants after the ideation phases of the experiment: participants rated the far analogical stimuli as significantly less relevant to the design problem than the near analogical stimuli $(F$ ( 1 , $44)=4.72, p=0.035, d=-0.64$; see Figure 3$)$. This was a composite measure, calculated by averaging ratings across two Likert scale questions from the survey: "This device is similar to devices that generate energy from human motion", and "The mechanisms in this device are relevant to designing devices that generate energy from human motion."

The results found in the study presented here are compared with the results found in a previous recent study by the authors [66], which examined the effect of analogical distance, commonness, and modality of external analogical stimuli on ideation performance. In that study, the experimental procedure, design problem, and metrics used to measure ideation performance were nearly identical to those used in the current study. The only discrepancies were that feature transfer and variability in design metrics were not coded for the current study due to a lower total number of ideas generated. The "near" stimuli were defined as "within domain" technologies, being patents describing energy generation technologies that could be used for analogical transfer to the human motion energy generation design problem. The "far" stimuli were defined as "cross domain technologies, or rather patents that described technologies that were not pertaining to energy/electricity generation, but could still potentially be used for analogical transfer to the design problem. These patents were chosen by mechanical engineering design faculty and a mechanical engineering design $\mathrm{PhD}$ candidate. This previous work indicated that far-field analogical stimuli were correlated with significantly better ideation performance over the near-field analogies in terms of novelty and quality. An exploration of the reconciliation of this previous study and the current study stimulates interesting, validating, and thought provoking discussion.
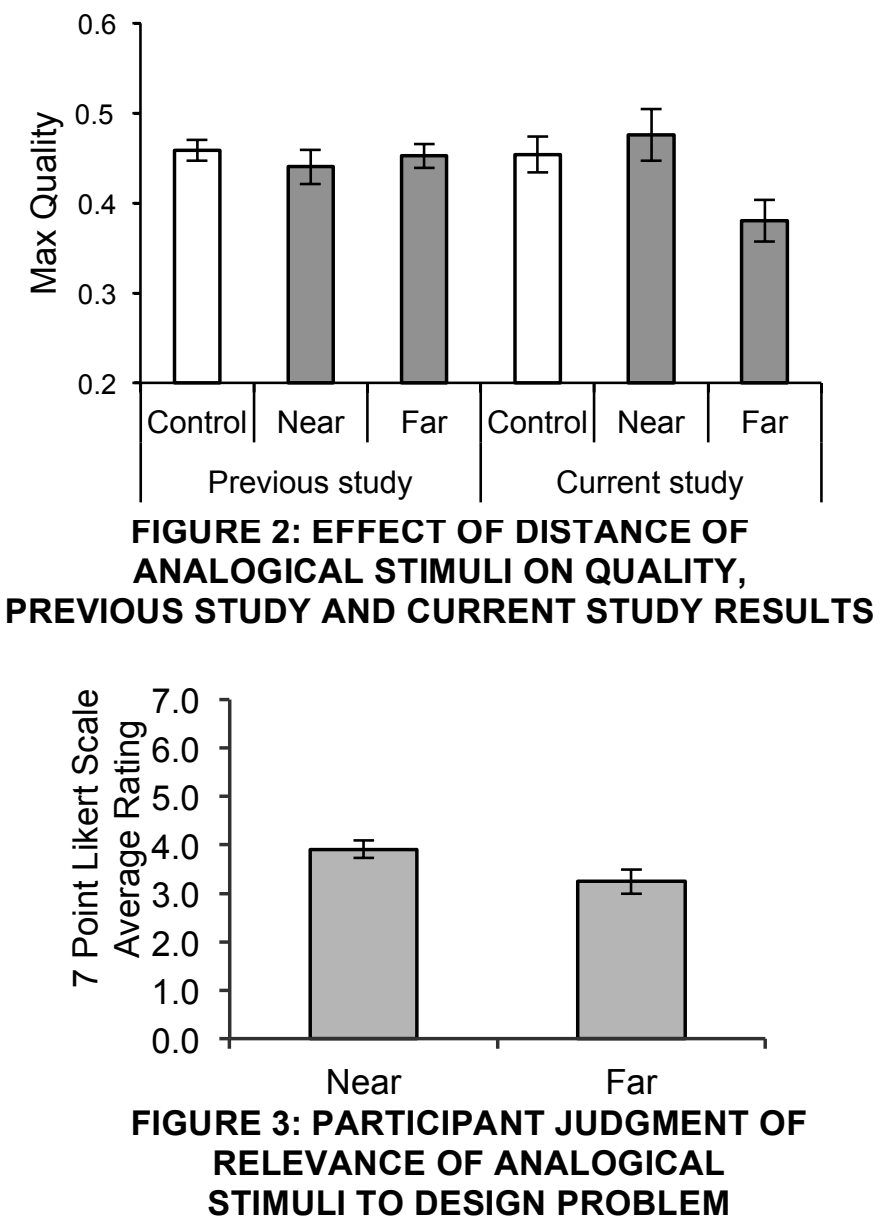

5 DISCUSSION

As indicated by the results presented in Section 4, it is encouraging to recognize that, in the current study, participants exposed to "near" patents were not significantly worse than the control condition participants in terms of novelty and quality of their design output - meaning that the external stimuli, though causing a greater cognitive load on the participants, was not detrimental to their performance on the design task. Further, the results in Section 4 
indicate that the patents designated as "near" patents in this study were significantly more helpful to designers during their ideation than the patents designated as "far" in terms of their effect on novelty and quality of design output, and in their perceived relevance to the design problem.

Figures 1 and 2, displaying the novelty and quality results from the previous and current studies, suggest what appear to be opposing conclusions regarding whether "near" or "far" patents are more beneficial to the design output. These seemingly opposing results raised the question: how consistently are the labels "near" and "far" used, not only in these two studies, but in the literature as a whole? Unfortunately, "near" and "far" when talking about the distance of analogies often mean something different to each researcher, and to each individual study or discussion. This makes generalization about the effects of distance of analogy on design and ideation a difficult task. To reconcile the findings of our two studies, we took advantage of the same process and tool used to choose the stimuli in the most recent work in this paper to understand how our definitions of "near" and "far" compared to one another.

Figure 4 displays the hierarchy structure created and used to choose the near and far patents for this study. All patent index numbers and corresponding U.S. patent database numbers can be found in Appendix A. The text of the design problem was used within LSA to find the effective "starting point" within the structure, chosen by calculating which node was most semantically similar to the design problem text. As stated in the Methodology Section, the near patents were chosen from the set of 45 if they were zero or one node away from the starting point. The far patents were chosen to be 3 nodes away from the starting point.

The 8 patents, 4 "near" and 4 "far" from the previous study were added to the structure of 45 random patents used to choose stimuli for this study. The resulting hierarchy structure is shown in Figure 5. Patents numbered 46-49 were "far" patents and those numbered 50-53 were "near" patents from the previous study.

It is interesting to find that all 8 patents from the previous study

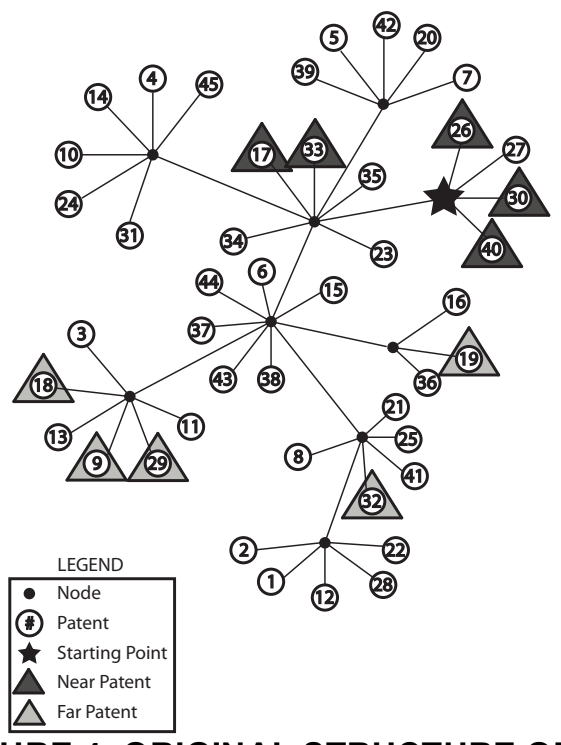

FIGURE 4: ORIGINAL STRUCTURE OF 45 RANDOM PATENTS USED TO CHOOSE STIMULI SETS IN THE CURRENT STUDY clustered closely, within zero or one node, around the design problem "starting point", with the "near" patents from the previous study being an average of 0 nodes away from the starting point and the "far" patents from the previous study being an average of 0.5 nodes away from the starting point; the patents designated as "near" in the current study were placed one or two nodes away from the starting point, an average of 1.4 nodes away (and thus farther away than the near patents from the first study); and the patents designated as "far" in this work were now placed, in all but one case, three or four nodes away from the starting point, with an average of 2.8 nodes away. This structure quantifies the relative distance of the external analogical stimuli to the design problem starting point, for two different studies. The handpicked near patents from the previous study are the closest set of patents to the design problem in the structure. The hand picked far patents from the previous study are still closer than the near patents chosen by the algorithm from the current study. This relative distance relationship makes sense in that the likelihood of having truly useful patents from only a random selection of 45 is small. As the better analogies are added to the mix, the set of randomly selected patents spreads out from the design problem. The relative distances in number of nodes in each distinct structure should be viewed not as an absolute measure but as a means for qualitative comparison - as the space and meaning of distance in the structures will change as the particular patents and number of patents within them change.

These results support our argument that near and far can have distinctly different meanings across the literature. In addition, it is a validation of the structuring methodology and its ability to portray relative analogical distance. This analysis method can be taken one step further, by adding yet another 100 random patents to the space to see how the relationships might change in an even larger context pool, better mimicking how these patents might be situated in the entire patent database. The resulting structure is shown in Figure 6.

The hierarchy structure in Figure 6 shows similarities to that in Figure 5, but with some interesting differences. The 8 patents from the previous work remain closely clustered around the design problem starting point, with the "near" patents being an average of 0.5 nodes away and the "far" patents being an average of 1.5 nodes away.

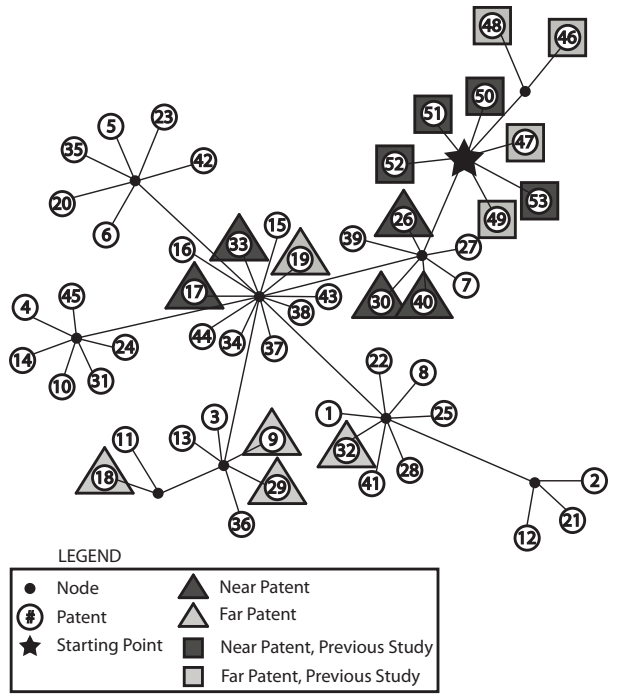

FIGURE 5: ORIGINAL STRUCTURE OF 45 RANDOM PATENTS WITH 8 PATENTS FROM PREVIOUS WORK 
However, the patents from the study presented in this paper are scattered in the structure in a more unexpected way. The "near" patents are now an average of 4.4 nodes away from the design problem suggest can be helpful to design inspiration [20], in that random analogical stimuli may be too distant from the design problem to allow for mapping or transfer to occur. The current study explored the

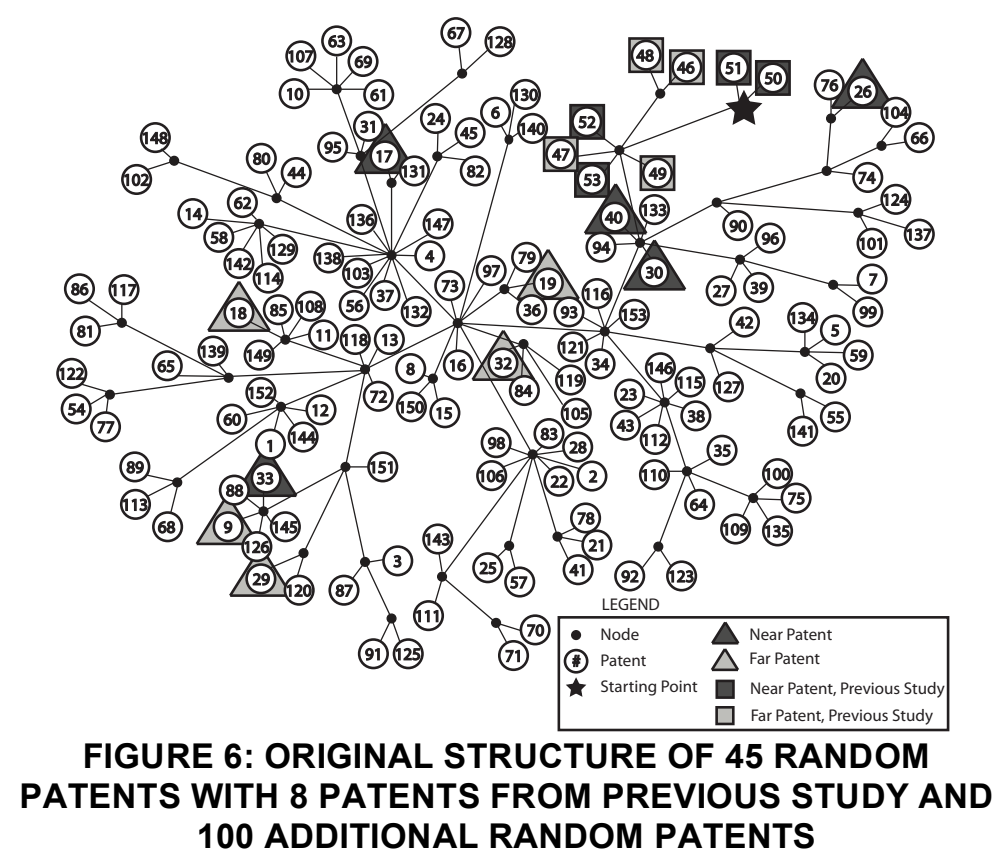

starting point, and the "far" patents are further out at an average of 6 nodes away; but in many cases, the patents from this work called "near" are found in the same cluster as those called "far", or are found the same distance away from the starting point. In a few cases, "far" patents are even found closer to the starting point than "near" patents.

It is hypothesized that the larger the structures become, the more well-tuned the structure will be, much the same to the logic behind using large sample sizes. As the number of patents that are included in the representative space and subsequent structure increases, the clustering, connections, and associations represented within the structure will become more meaningful, as there are more likely to be useful patents for analogical stimulation. With this hypothesis in mind, we focus discussion on the structure in Figure 6 . The results in the previous study imply that "far" patents as analogical stimuli are more beneficial than "near" patents to design output quality. The structure in Figure 6 shows that the "near" patents from the previous study are closer to the design problem in semantic similarity than the "far" patents. The results from the current study imply that "near" patents are more beneficial than "far" patents to design output quality. The "near" patents from the current study are closer to the design problem starting point than the "far" patents from the current study, but not as near as the "far" patents from the previous study.

These results taken all together suggest that there is a "sweet spot" for distance from the design problem when choosing analogical stimuli - in other words, there may be such a thing as "too near" and "too far" when searching for analogies to employ in design by analogy ideation practice. The "near" patents from the previous study were too "near" to be beneficial to designers as analogical stimuli. The "far" patents from the current study were too "far" to be useful to the designers, as well. This conclusion is reinforced by the finding presented in Figure 3, showing that the participants found the "far" patents to be significantly less relevant to the design problem. Our findings directly challenge the random inspiration methods that some

impact of example solutions on novice designers, arguing for a sweet spot for analogical distance to the given design problem. Based on previous work showing that experts do use analogies but can be fixated by those that include very near or close examples [74], we expect that a similar sweet spot exists, possibly with different parameters that should be explored in future work. It is important to note that this suggested "sweet spot" is dependent upon and specific to the patents and design problem, and number of patents used in this study, and would change as these variables change. The stabilization of this sweet spot is an area for future work.

The results presented here are impactful for a few reasons. First, there is promise for the use of this structuring technique as the basis of a design inspiration tool for automatically finding design analogies. Designers often employ design-by-analogy through coming up with the analogies themselves, through a stroke of luck, low hanging fruit, or genius. The current most widely used method for searching for analogical inspiration in the patent database is through key word search. The results of key word searches can be an overwhelming undertaking to explore for design inspiration. There are also computational "innovation support tools" for sale to businesses and innovators [75]. All of these methods and tools place the onus largely on designers to generate the terms or analogies of their own accord and comb through search results. It is known in the psychology literature that the retrieval of far-field analogies is cognitively difficult [23]. In addition, remindings tend to be constrained by surface similarity [24], meaning the probability of retrieving surface dissimilar analogies is low. Thus, a computational design tool that could find analogies in the "sweet spot" that is indicated from the work presented here, which would not easily be located by a designer due to surface dissimilarity or rarity of occurrence, could be exceedingly helpful to facilitating the practical use of the design-by-analogy method. With a large population of patents to build the structures, designers could have fast and relatively easy access to relevant analogies that could be useful or 
inspirational to them, that they may not have otherwise thought of or been able to find.

Future work includes extending this methodology to create very large, descriptive structures of the patent database to use as a tool in cognitive studies to explore the design space in a methodical, quantified way. One particular avenue of extension would take advantage of the potential information contained in the structuring and clustering of the patents that goes beyond simply delineating potential relevance to the design problem. For example, the grouping of patents into nodes based on functional similarity could highlight features and functional principles that would otherwise potentially be overlooked (e.g., via analogical comparison; $[76,77]$ ) yielding fresh insights for creative ideation. In the cognitive psychology literature, it has been shown that enabling changes in representation of objects and/or ideas, for example by leading problem solvers to attend to previously ignored features, is an effective way of dealing with "functional fixedness", where problem solvers have difficulty seeing a potential creative use of an object with which they are familiar [78, 79]. Labeling of nodes or entities in these structures could also facilitate multiple representations of potentially relevant functional principles for designby-analogy.

The broader goal of this work is to reach a robust cohesive theory of the ways in which analogical stimuli could be most optimally structured and prepared for exploration to support creative design ideation.

\section{CONCLUSIONS}

The cognitive engineering design experiment presented in this paper, combined with those of the previous study performed by the authors, suggest that the terms "near" and "far" when referring to distance of analogies are contextual and relative terms. The nature of the use of these terms in the literature makes it difficult to formulate a cohesive theory of analogical distance and its effect on design output quality. The analysis presented in this paper takes a step toward reconciling diverse findings. The use of a structuring method combining Latent Semantic Analysis and a hierarchical Bayesian algorithm for choosing and analyzing the analogical stimuli in both studies suggests that there is a "sweet spot" for distance of analogies, and that there is such a thing as too "near" and too "far" in design analogies. The structuring method presented shows promise as a facilitator for a unified methodology and eventual development of a cohesive and robust theory on the effect of distance of analogy on design output, as well as providing a basis for an automated design analogy finding tool for supporting creative design ideation.

\section{ACKNOWLEDGMENTS}

This work is supported by the National Science Foundation, under the grant: CMMI0855326.

\section{REFERENCES}

[1] H. Casakin and G. Goldschmidt, "Expertise and the use of visual analogy: implications for design education," Design Studies, vol. 20, no. 2, pp. 153-175, Mar. 1999.

[2] A. K. Goel, "Design, analogy, and creativity," IEEE Expert, vol. 12, no. 3, pp. 62-70, 1997.

[3] B. T. Christensen and C. D. Schunn, "The relationship of analogical distance to analogical function and preinventive structure: the case of engineering design.," Memory \& Cognition, vol. 35, no. 1, pp. 29-38, Jan. 2007.

[4] J. Linsey, J. Murphy, J. Laux, A. B. Markman, and K. L. Wood, "Supporting innovation by promoting analogical reasoning," in Tools for innovation, A. B. Markman and K. L. Wood, Eds. New York, NY: Oxford University Press, 2009.

[5] K. Fu, J. Cagan, K. Kotovsky, and K. L. Wood, "Discovering Structure In Design Databases Through Functional And Surface Based Mapping," in Proceedings of the ASME DTM Conference, 2011.

[6] K. Fu, J. Cagan, and K. Kotovsky, "A methodology for discovering structure in design databases," in Proceedings of the 18th International Conference on Engineering Design (ICED11), 2011.

[7] I. Tseng, J. Moss, J. Cagan, and K. Kotovsky, "The role of timing and analogical similarity in the stimulation of idea generation in design," Design Studies, vol. 29, no. 3, pp. 203-221, May 2008.

[8] J. Moss, K. Kotovsky, and J. Cagan, "The influence of open goals on the acquisition of problem-relevant information," Journal of Experimental Psychology: Learning, Memory, and Cognition, vol. 33, no. 5, pp. 87691, Sep. 2007.

[9] J. Moss, J. Cagan, and K. Kotovsky, "Design Ideas and Impasses: The Role of Open Goals," in Proceedings of the 16th International Conference on Engineering Design (ICED07), 2007.

[10] J. S. Linsey, K. L. Wood, and A. B. Markman, "Modality and representation in analogy," Artificial Intelligence for Engineering Design, Analysis and Manufacturing, vol. 22, no. 2, pp. 85-100, Mar. 2008.

[11] J. Hey, J. Linsey, A. M. Agogino, and K. L. Wood, "Analogies and Metaphors in Creative Design," International Journal of Engineering Education, vol. 24, no. 2, p. 12, 2008.

[12] A. B. Markman, K. L. Wood, J. Linsey, J. Murphy, and J. Laux, "Supporting innovation by promoting analogical reasoning," 2011.

[13] S. Vattam, M. Helms, and A. Goel, "A Content Account of Creative Analogies in Biologically Inspired Design," AIEDAM, Special Issue on Biologically Inspired Design, 24: 467-481, 2010.

T. Taura, Y. Nagai, and S. Tanaka, "Design Space Blending - A Key For Creative Design," Proceedings of ICED, Melbourne, Australia, August 15-18, 2005.

K. Koile, “An Intelligent Assistant For Conceptual Design: Informed Search Using A Mapping Of Abstract Qualities To Physical Form", Proceedings of Design Computing and Cognition '04, 3-22, 2004. W. Visser, 1996, "Two functions of analogical reasoning in design: A cognitive-psychology approach”, Design Studies, Special Issue on Design Cognition and Computation, 17, 417-434, 1996.

D. Gentner and A. B. Markman, "Structure mapping in analogy and similarity," American Psychologist, vol. 52, no. 1, pp. 45-56, 1997. D. W. Dahl and P. Moreau, "The Influence and value of analogical thinking during new product ideation," Journal of Marketing Research, vol. 39 , no. 1 , pp. $47-60,2002$.

J. O. Wilson, D. Rosen, B. A. Nelson, and J. Yen, "The effects of biological examples in idea generation," Design Studies, vol. 31, no. 2, pp. 169-186, Mar. 2010.

J. H. Dyer, H. B. Gregersen, and C. M. Christensen, The Innovator's DNA: Mastering the Five Skills of Disruptive Innovators. Boston, MA: Harvard Business Review Press, 2011.

K. N. Dunbar, "How scientists think: On-line creativity and conceptual change in science," in Creative thought: An investigation of conceptual structures and processes, T. B. Ward, S. M. Smith, and J. Vaid, Eds. Washington D.C.: American Psychological Association, 1997, pp. 461493.

R. W. Weisberg, "On 'out-of-the-box' thinking in creativity," in Tools for innovation, A. Markman and K. Wood, Eds. New York, NY: Oxford University Press, 2009, pp. 23-47.

[23] M. L. Gick and K. J. Holyoak, “Analogical problem solving,” Cognitive Psychology, vol. 12, no. 3, pp. 306-355, 1980.

K. D. Forbus, D. Gentner, and K. Law, "MAC/FAC: A model of similarity-based retrieval," Cognitive Science, vol. 19, pp. 141-205, 1994.

W. J. J. Gordon, Synectics: The Development of Creative Capacity. New York, NY: Harper and Brothers, 1961.

M. French, Invention and Evolution: Design in Nature and Engineering. Cambridge, UK: Cambridge University Press, 1988.

J. Linsey, A. B. Markman, and K. L. Wood, "Design by Analogy: A Study of the WordTree Method for Problem Re-Representation," Journal of Mechanical Design, 2012.

J. Linsey, A. B. Markman, and K. L. Wood, "WordTrees: A method for design-by-analogy," in Proceedings of the 2008 ASEE Annual Conference, 2008. 
[29] J. S. Linsey, K. L. Wood, and A. B. Markman, "Increasing innovation: Presentation and evaluation of the WordTree design-by-analogy method," in Proceedings of the 2008 IDETC/CIE Conference, 2008.

[30] E. Hacco and L. H. Shu, "Biomimetic concept generation applied to design for remanufacture," in ASME IDETC/CIE, 2002.

[31] D. A. McAdams and K. L. Wood, "Quantitative measures for design by analogy," in DETC '00, 2000 ASME IDETC/CIE 2000.

[32] J. Hirtz, R. B. Stone, D. A. Mcadams, S. Szykman, and K. L. Wood, “A functional basis for engineering design: Reconciling and evolving previous efforts," Research in Engineering Design, 13, pp. 65-82, 2002.

[33] K. Rantanen and E. Domb, Simplified TRIZ: New Problem Solving Applications for Engineers and Manufacturing Professionals, 2nd ed. New York, NY: Auerbach Publications, 2007.

[34] R. Zhang, J. Cha, and Y. Lu, "A conceptual design model using axiomatic design, functional basis and TRIZ," in Proceedings of the 2007 IEEE International Conference on Industrial Engineering and Engineering Management, 2007.

[35] A. K. Chakrabarti, I. Dror, and N. Eakabuse, "Interorganizational transfer of knowledge: an analysis of patent citations of a defense firm," IEEE Transactions on Engineering Management, 40(1), 91-94, 1993.

[36] K. V. Indukuri, A. A. Ambekar, and A. Sureka, "Similarity Analysis of Patent Claims Using Natural Language Processing Techniques," in Proceedings of International Conference on Computational Intelligence and Multimedia Applications (ICCIMA 2007), 2007, pp. 169-175.

[37] K. Kasravi and M. Risov, "Patent Mining - Discover y of Business Value from Patent Repositor ies," in Proceedings of 2007 40th Annual Hawaii International Conference on System Sciences (HICSS'07), 2007, 54-54.

[38] M. R. Bohm, J. P. Vucovich, and R. B. Stone, "Capturing Creativity: Using a Design Repository to Drive Concept Innovation," in Proceedings of ASME IDETC, DETC05/CIE-85105, 2005, vol. 2005, no. 47403.

[39] S. Koch, H. Bosch, M. Giereth, and T. Ertl, "Iterative Integration of Visual Insights during Scalable Patent Search and Analysis.," in Proceedings of IEEE Transactions on Visualization and Computer Graphics, 2009.

[40] J. T. Murphy, "Patent-based analogy search tool for innovative concept generation," Ph.D. Dissertation, The University of Texas, Department of Mechanical Engineering, 2011.

[41] S. Mukherjea, B. Bamba, and P. Kankar, "Information Retrieval and Knowledge Discovery Utilizing a BioMedical Patent Semantic Web," IEEE Transactions on Knowledge and Data Engineering, vol. 17, no. 8, pp. 1099-1110, 2005.

[42] S. Chakrabarti, B. Dom, R. Agrawal, and P. Raghavan, "Scalable feature selection, classification and signature generation for organizing large text databases into hierarchical topic taxonomies," The VLDB Journal, vol. 7, no. 3, pp. 163-178, Aug. 1998

[43] T. L. Griffiths, C. Kemp, and J. B. Tenenbaum, "Bayesian models of cognition," in Cambridge Handbook of Computational Psychology, R. Sun, Ed. New York, NY: Cambridge University Press, 2008, pp. 59-100.

[44] C. Kemp and J. B. Tenenbaum, "The discovery of structural form," Proceedings of the National Academy of Sciences, vol. 105, no. 31, pp. 10687-10692, Aug. 2008.

[45] R. N. Shepard, "Multidimensional scaling, tree-fitting, and clustering.," Science, vol. 210, no. 4468, pp. 390-398, Oct. 1980.

[46] B. Inhelder and J. Piaget, The Early Growth of Logic in the Child. New York, NY: W W Norton \& Company, 1969.

[47] J. R. Anderson, "The adaptive nature of human categorization," Psychological Review, vol. 98, no. 3, pp. 409-429, 1991.

[48] J. P. Huelsenbeck and F. Ronquist, "MRBAYES: Bayesian inference of phylogenetic trees.," Bioinformatics, vol. 17, 8, pp. 754-755, Aug. 2001

[49] A. P. Fiske, "The four elementary forms of sociality: framework for a unified theory of social relations.," Psychological Review, vol. 99, no. 4, pp. 689-723, Oct. 1992.

[50] L. Guttman, "A basis for scaling qualitative data," American Sociological Review, vol. 9, no. 2, pp. 139-150, 1944.

[51] R. A. Bradley and M. E. Terry, "Rank analysis of incomplete block designs. 1. The method of paired comparisons," Biometrika, vol. 39, pp. 324-345, 1952

[52] L. Guttman, "A new approach to factor analysis: The Radex," in Mathematical Thinking in the Social Sciences, P. F. Lazarsfeld, Ed. New York, NY: Free Press, 1954, pp. 258-348.

[53] J. S. Wiggins, "An informal history of the interpersonal circumplex tradition," Journal of Personality Assessment, 66, 2, 217-33, Apr. 1996.

[54] P. H. Sneath and R. R. Sokal, Numerical Taxonomy: The Principles and Practice of Numerical Classification. San Francisco: Freeman, 1973.
A. M. Collins and M. R. Quillian, "Retrieval time from semantic memory," Journal of Verbal Learning and Verbal Behavior, vol. 8, no. 2, pp. 240-247, Apr. 1969.

J. D. Carroll, "Spatial, nonspatial and hybrid models for scaling,"

Psychometrika, vol. 41, pp. 439-463, 1976.
T. Kohonen, Self-Organizing Maps. New York, NY: Springer, 1997. T. K. Landauer and S. T. Dumais, “A solution to Plato's problem: The latent semantic analysis theory of acquisition, induction, and representation of knowledge," Psychological Review, vol. 1, no. 2, pp. 211-240, 1997.

$$
\text { A. M. Agogino, S. Song, and J. Hey, "Triangulation of Indicators of }
$$

Successful Student Design Teams," International Journal of Engineering Education, vol. 22, no. 3, pp. 617-625, 2006.

A. Dong and A. M. Agogino, "Text Analysis for Constructing Design Representations," Artificial Intelligence in Engineering, 11, 65-75, 1997. J. Moss, K. Kotovsky, and J. Cagan, "The role of functionality in the mental representations of engineering students: some differences in the early stages of expertise," Cognitive Science, 30, 1, pp. 65-93, Jan. 2006. T. K. Landauer, P. W. Foltz, and D. Laham, "An introduction to latent semantic analysis," Discourse Processes, vol. 25, no. 2, pp. 259-284, 1998.

P. W. Foltz, W. Kintsch, and T. K. Landauer, "The measurement of textual coherence with latent semantic analysis," Discourse Processes, vol. 25, no. 2-3, pp. 285-307, Jan. 1998.

S. Deerwester, S. T. Dumais, G. W. Furnas, and T. K. Landauer, "Indexing by Latent Semantic Analysis.," Journal of the American Society for Information Science, vol. 41, no. 6, p. 1990, 1990. C. Kemp and Tenenbaum, "The discovery of structural form," Proceedings of the National Academy of Sciences, Supporting Information Appendix, 2008.

J. Chan, K. Fu, C. D. Schunn, J. Cagan, K. L. Wood, and K. Kotovsky, "On the benefits and pitfalls of analogies for innovative design: Ideation performance based on analogical distance, commonness, and modality of examples," Journal of Mechanical Design, vol. 133, p. 081004, 2011. M. Green, A. Duston, K. L. Wood, R. Stone, and D. McAdams, "Integrating Service-Oriented Design Projects in the Engineering Curriculum," in Proceedings of the ASEE Annual Conference and Exposition 2002, 2002.

M. Green and K. L. Wood, "Service-Learning Approaches to International Humanitarian Design Projects: Assessment of Spiritual Impact," in Proceedings of the 2004 Christian Engineering Education Conference, 2004.

C. White and K. L. Wood, "Influences and Interests in Humanitarian Engineering," in Proceedings of the 2010 ASEE Annual Conference, Global Colloqium on Engineering Education, 2010.

A. B. Markman and K. L. Wood, Tools for innovation. New York, NY:

Oxford University Press, 2009.
M. A. Boden, The creative mind: Myths and mechanisms. New York: Routledge, 2004.

J. J. Shah, N. Vargas-Hernandez, and S. M. Smith, "Metrics for measuring ideation effectiveness," Design Studies, vol. 24, no. 2, pp. 111-134, Mar. 2003.

K. Girotra, C. Terwiesch, and K. T. Ulrich, "Idea generation and the quality of the best idea," Management Science, 56, pp. 591-605, 2010. J. S. Linsey, I. Tseng, K. Fu, J. Cagan, K. L. Wood, and C. D. Schunn, "A Study of Design Fixation, Its Mitigation and Perception in Engineering Design Faculty," Journal of Mechanical Design, vol. 132, no. 4, p. 041003, Apr. 2010.

"Invention Machine Goldfire: Unleashing the Power of Research," Invention Machine Corporation, Accessed 19 February 2012, http://inventionmachine.com/products-and-services/innovationsoftware/goldfire-Research/.

D. Gentner, S. Brem, R. W. Ferguson, P. Wolff, A. B. Markman, and K. D. Forbus, "Analogy and Creativity in the Works of Johannes Kepler," in Creative thought: An investigation of conceptual structures and processes, T. B. Ward, S. M. Smith, and J. Vaid, Eds. Washington D.C.: American Psychological Association, 1997, pp. 403-459.

K. Kurtz, C. Miao, and D. Gentner, "Learning by Analogical Bootstrapping," Journal of the Learning Sciences, vol. 10, no. 4, pp. 417-446, Oct. 2001.

C. Kaplan and H. A. Simon, "In search of insight," Cognitive Psychology, vol. 22, no. 3, pp. 374-419, 1990.

T. McCaffrey, "Innovation Relies on the Obscure: A Key to Overcoming the Classic Problem of Functional Fixedness," Psychological Science, vol. 23 , no. 3 , pp. $215-218,2012$. 


\begin{tabular}{|c|c|}
\hline $\begin{array}{c}\text { Patent } \\
\text { Index }\end{array}$ & $\begin{array}{c}\text { U.S. Patent } \\
\text { Number }\end{array}$ \\
\hline 1 & $5,626,594$ \\
\hline 2 & $3,945,652$ \\
\hline 3 & $6,264,019$ \\
\hline 4 & $6,298,842$ \\
\hline 5 & $5,343,936$ \\
\hline 6 & $6,300,699$ \\
\hline 7 & $4,400,154$ \\
\hline 8 & $4,078,716$ \\
\hline 9 & $5,234,096$ \\
\hline 10 & $4,780,075$ \\
\hline 11 & $5,988,780$ \\
\hline 12 & $5,542,526$ \\
\hline 13 & $5,107,608$ \\
\hline 14 & $6,543,101$ \\
\hline 15 & $6,398,066$ \\
\hline 16 & $4,026,453$ \\
\hline 17 & $6,685,268$ \\
\hline 18 & $4,508,361$ \\
\hline 19 & $5,085,240$ \\
\hline 20 & $5,171,128$ \\
\hline 21 & $6,551,037$ \\
\hline 22 & $4,343,498$ \\
\hline 23 & $5,165,473$ \\
\hline 24 & $4,232,428$ \\
\hline 25 & $3,963,052$ \\
\hline 28 & $5,018,908$ \\
\hline 29 & $5,277,276$ \\
\hline 30 & $6,109,307$ \\
\hline 31 & $6,666,211$ \\
\hline
\end{tabular}

\begin{tabular}{|l|l|}
\hline 32 & $6,082,923$ \\
\hline 33 & $6,453,834$ \\
\hline 34 & $4,440,105$ \\
\hline 35 & $4,389,922$ \\
\hline 36 & $5,378,106$ \\
\hline 37 & $4,950,250$ \\
\hline 38 & $4,050,047$ \\
\hline 39 & $5,452,860$ \\
\hline 40 & $5,160,091$ \\
\hline 41 & $5,377,379$ \\
\hline 42 & $4,162,046$ \\
\hline 43 & $4,380,216$ \\
\hline 44 & $6,669,716$ \\
\hline 45 & $3,977,562$ \\
\hline 46 & $4,335,611$ \\
\hline 47 & $4,402,483$ \\
\hline 48 & $4,568,937$ \\
\hline 49 & $4,139,981$ \\
\hline 50 & $4,266,143$ \\
\hline 51 & $4,247,785$ \\
\hline 52 & $6,208,037$ \\
\hline 55 & $4,506,651$ \\
\hline 56 & $5,239,707$ \\
\hline 57 & $4,535,756$ \\
\hline 58 & $4,304,332$ \\
\hline 59 & $6,612,806$ \\
\hline 60 & $5,762,169$ \\
\hline 61 & $6,716,115$ \\
\hline 62 & $4,678,083$ \\
\hline 63 & $4,813,672$ \\
\hline 64 & $4,649,970$ \\
\hline & \\
\hline 50
\end{tabular}

APPENDIX

PATENT INDEX

\begin{tabular}{|l|l|}
\hline 65 & $6,481,735$ \\
\hline 66 & $4,380,233$ \\
\hline 67 & $6,991,656$ \\
\hline 68 & $7,059,508$ \\
\hline 69 & $4,488,547$ \\
\hline 70 & $5,228,241$ \\
\hline 71 & $4,379,706$ \\
\hline 72 & $4,233,773$ \\
\hline 73 & $5,416,955$ \\
\hline 74 & $7,215,986$ \\
\hline 75 & $4,705,065$ \\
\hline 76 & $4,432,481$ \\
\hline 77 & $4,203,505$ \\
\hline 78 & $3,970,149$ \\
\hline 79 & $5,590,608$ \\
\hline 82 & $5,768,928$ \\
\hline 83 & $6,044,919$ \\
\hline 84 & $5,921,843$ \\
\hline 85 & $4,483,066$ \\
\hline 86 & $6,776,447$ \\
\hline 87 & $4,913,681$ \\
\hline 88 & $5,964,159$ \\
\hline 89 & $6,505,991$ \\
\hline 90 & $6,616,409$ \\
\hline 91 & $6,769,593$ \\
\hline 92 & $6,782,855$ \\
\hline 93 & $4,251,075$ \\
\hline 94 & $5,528,878$ \\
\hline 95 & $5,819,391$ \\
\hline 96 & $5,842,652$ \\
\hline 97 & $4,407,173$ \\
\hline
\end{tabular}

\begin{tabular}{|l|l|}
\hline 131 & $5,273,173$ \\
\hline 132 & $5,438,724$ \\
\hline 133 & $6,974,456$ \\
\hline 135 & $3,941,514$ \\
\hline 136 & $6,109,282$ \\
\hline 137 & $4,303,397$ \\
\hline 138 & $5,899,571$ \\
\hline 139 & $6,234,452$ \\
\hline 140 & $4,841,621$ \\
\hline 141 & $4,142,679$ \\
\hline 142 & $6,634,044$ \\
\hline 143 & $4,270,310$ \\
\hline 144 & $5,423,097$ \\
\hline 145 & $5,572,898$ \\
\hline 146 & $3,938,909$ \\
\hline 147 & $5,647,066$ \\
\hline 148 & $6,119,041$ \\
\hline 149 & $4,484,762$ \\
\hline 150 & $4,762,262$ \\
\hline 151 & $6,164,698$ \\
\hline 152 & $6,062,856$ \\
\hline 153 & $4,739,727$ \\
\hline
\end{tabular}

